\title{
Automatic Object Recognition from Satellite Images using Artificial Neural Network
}

\author{
Anil Kumar Goswami \\ DRDO \\ Metcalfe House, Delhi
}

\author{
Shalini Gakhar \\ PDM College of Engg. for Women \\ Bahadurgarh, Haryana
}

\author{
Harneet Kaur \\ PDM College of Engg. for Women \\ Bahadurgarh, Haryana
}

\begin{abstract}
Object recognition from satellite images is a very important application for various purposes. Objects can be recognized based on certain features and then applying some algorithm to extract those objects. Basically object recognition is a classification problem. For various remote sensing applications, waterbody acts as an important object which needs to be extracted. It is wise and better if possible, to extract waterbody object automatically from satellite data without any human intervention. This can be achieved using machine learning techniques. Artificial Neural Network (ANN) is such technique which makes machine intelligent by providing learning to it. This intelligent machine can extract objects automatically. This paper presents a methodology to extract waterbody object from satellite data in an automatic manner with the help of ANN. Training and testing dataset have been created by a domain expert which then have been used to train Multi Layer Perceptron (MLP) using Error Back Propagation (EBP) learning algorithm. Confusion matrix and Kappa coefficient have been used for accuracy assessment.
\end{abstract}

\section{General Terms}

Object Recognition, Satellite Images, Machine Learning, Image Classification, Feature Extraction, Kappa Coefficient, Confusion Matrix, Training and Testing Datasets

\section{Keywords}

Object Recognition, Artificial Neural Network (ANN), Multi Layer Perceptron (MLP), Error Back Propagation (EBP), Waterbody, Automatic Waterbody Extraction, Weight File, Image Classification, Supervised Classification

\section{INTRODUCTION}

Satellite images are one of useful resources to extract various types of information for many remote sensing and image processing applications. One of the important applications is object recognition. Objects are extracted based on certain features using various algorithms. This extraction of objects can be (1) manual i.e. by domain expert or (2) automatic i.e. with the help of machine learning. Machine Learning means providing learning to machines to make them intelligent. A learned machine can automatically recognize objects of interest. One of algorithm used to achieve machine learning is Artificial Neural Network (ANN). ANN can be Single Layer Perceptron (SLP) or Multi Layer Perceptron (MLP). MLP is a Feedforward ANN consisting of many hidden layers and works on certain sets of input data to generate a set of outputs. It makes use of Error Back Propagation (EBP) algorithm to learn a particular set of data and thus solving the problem. The satellite images are having various natural objects in isolation or combination such as mountains, forests, vegetations, plains, plateaus, water bodies and many more. Waterbody is an important object that needs to be extracted automatically from satellite images. Automatic extraction of waterbody becomes a key component in order to assess the existing water resource and help in planning for various purposes.

This paper provides a framework for automatic extraction of waterbody object from satellite data by using machine learning with the help of ANN. This paper has been divided into six sections. Second section is literature survey that enlightens the work done in this area. Third section is theoretical framework providing details of theory behind the experiment. This section is followed by methodology that describes detailed design and implementation of the experiment. The next section presents the results and discussions for the experiment. The last section gives the conclusion.

\section{LITERATURE SURVEY}

W.S. McCulloch, W. Pits described the first Neural Network Model and F. Rosenblatt (Perceptron) and B. Widrow (Adaline) developed the first training algorithm [1]. A neural network is a massively parallel distributed processor that has a natural propensity for storing experiential knowledge and making it available for use. It resembles the brain in two respects [2]. Firstly, Knowledge is acquired by the network through a learning process and secondly, interneuron connection strengths known as synaptic weights are used to store the knowledge. Artificial neural systems, or neural networks, are physical cellular systems which can acquire, store, and utilize experiential knowledge [3]. The most well known types of ANN are: (a) Feedforward ANN: In a Feedforward ANN, the connections between units do not form cycles. It usually produces a response to an input quickly. The Feedforward MLP [4], [5] network is a widely used model in ANN using the back propagation algorithm [6], [7] for real world data. However, it is often avoided due to the large size of network and the training that would be too slow to be tolerable. (b) Feedback ANN: In a feedback or recurrent ANN, there are cycles in the connections. In some feedback NNs, each time an input is presented, the NN must iterate for a potentially long time before it produces a response. MLP has been a wide area for various applications, all of which can be stratified as pattern classification, function approximation or prediction. Pattern classification is the configuration of patterns into groups of patterns having the same set of properties. It is well known that MLPs are universal in the sense that they can approximate any continuous nonlinear function arbitrarily well on a compact interval. As a result MLP became popular in order to parameterize nonlinear models and classifiers, often leading to improved results compared to classical [4], [8] MLP has proved to be a very effective tool for the classification of remote-sensing images. But, the training of such a classifier, by using data with very different a priori class probabilities (imbalanced data), is very slow. It is an effective method which describes a learning 
technique aimed at speeding up the training of a MLP also the classification becomes stable (with respect to initial weights) when applied to imbalanced data [9]. Various efforts are being done in order to optimize the fault tolerance of MLP in pattern classification problems. Fault tolerance is a frequently cited advantage of ANN. SLP was considerably less fault tolerant than any of the MLPs, including one with fewer adjustable weights [10]. MLP has been applied within the field of airquality prediction. According to the work of Yi and Prybutok, MLP helped in predicting ozone concentration on the surface of an industrial area in North America [11]. Weather forecasting is a difficult task to be undertaken. In 1996 Marzban and Stumpf predicted the existence of tornadoes, using MLP. This approach outperformed other techniques including discriminant analysis, logistic regression and rule based algorithm [12]. MLP was used for many applications such as predicting monsoon and rainfall [13] distinguishing clouds and ice or snow in Polar Regions, [14] interpret satellite imagery for identifying cyclones, war fronts, and weather conditions [15]. MLP can act as a useful tool to implement various other applications such as: paper currency recognition, the diagnosis of low back pain and sciatica, heart disease and cancer, stock market prediction, prediction of daily global solar radiation, handwritten character recognition, image classification, object recognition, feature extraction and many more.

\section{THEORETICAL FRAMEWORK 3.1 Artificial Neural Network (ANN)}

ANN is a computational model that imitates the structure and functionality of biological neurons of human brain. ANN can be either SLP consisting of only one hidden layer or MLP consisting of more than one hidden layer. MLP consists of input layer, output layer and hidden layers. Each layer consists of many nodes. Nodes in layers are connected with each other as shown in Figure 1.

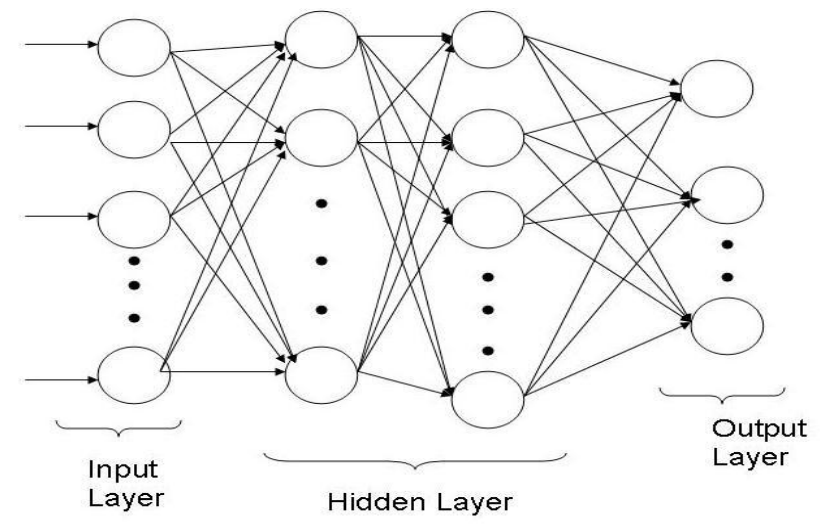

Figure 1: MLP Architecture

The inputs and the outputs of ANN are non-linearly dependent. Perception is the basic fundamental unit of ANN which is used for classification of patterns and was invented by Rosenblatt in 1958. Learning can be broadly divided into supervised and unsupervised techniques. MLP follows the Supervised Learning technique where prior information of desired response is known. Many algorithms are proposed till now to train MLP network. One of them is EBP algorithm. As per EBP the data flow is directed from the input layer to the output layer. The hidden layer acts as an intermediate computational unit between them. The input layer feeds the input data corresponding to each pattern into the network. The computation is done at each node of each layer in feed- forward manner. The computed output is compared with the actual output to provide the feedback that updates synaptic weights resulting into correction of computed output. So, there are three major part of EBP algorithm namely feed forward, compute gradient and update weights.

\subsection{Satellite Images}

A satellite image consists of collection of pixels arranged in matrix form i.e. rows and column. Each pixel can be represented by a vector of the size of number of bands. In case of multispectral data, there are 3 or 4 bands corresponding to each pixel. Satellite captures the data of area covered by them in form of reflectance values which are then converted into pixel values based on certain well established computation. These images are captured by various remote sensing satellites. Satellite images are having significant information with respect to natural objects. These images are used for various applications for civilian sector.

\subsection{Waterbody}

Waterbody is an important natural object and can be captured by remote sensing satellite. $70 \%$ of total Earth is covered with water. Out of which approx. $68 \%$ is saline water and is part of many water resources such as glaciers, oceans, sea, ponds etc. and only $2 \%$ is fit for drinking or portable water. Waterbody is an area or region with well defined topographical boundary where the water accumulates in large quantity, for example: river, ocean, sea, lake, reservoir etc. Water in some water bodies is either stagnant or flowing. Waterbody object can be identified by domain expert in a satellite image based on pixel values. For many applications, it is necessary to extract waterbody and its extent.

\subsection{Automatic Extraction of Waterbody Object}

As discussed above, waterbody is an important natural resource and this object can be recognized and extracted from satellite image based on various algorithms. A domain expert of remote sensing field can identify waterbody in a satellite image based on his experience and expertise. Although, there are many ways to extract essential natural objects from satellite image with the help of domain expert but such options are infeasible or costly in long term. It is also possible to extract waterbody object from satellite image in an automatic manner by using various algorithms. One of such algorithm is ANN as explained above. This paper presents a framework to extract waterbody object from satellite image in an automatic manner by using MLP architecture of ANN.

\section{METHODOLOGY}

This section describes the overall methodology of the software system developed and the experiment conducted to extract waterbody from satellite images based on ANN approach. ANN is a supervised classification approach. Hence, training and testing dataset have been created with respect to the problem of waterbody extraction by the domain expert. For ANN, MLP has been implemented and EBP algorithm has been used to provide training to the MLP. EBP is a well known algorithm for learning of ANN. The training dataset and MLP parameters i.e. Number of hidden layers, Nodes in each hidden layer, Mean Squared Error (MSE) threshold value, Learning rate and Coefficient of activation function are given as input to MLP for training process. EBP algorithm basically contains three modules namely feedforward, gradient computation and weight updation. These three modules are executed iteratively until global minima is achieved. Global minima is a point where MSE is minimum. 
Training of MLP is completed after global minima is reached. After completion of training of MLP, the resultant updated weights are stored as weight file which acts as memory for MLP working and MLP testing. After providing training to MLP, it is tested with testing dataset. The testing dataset and the weight file are given as input and feedforward is performed to get MLP output which is then compared with actual output in testing dataset to compute the testing accuracy. Both training and testing accuracy are computed with respect to training dataset and testing dataset respectively. This MLP has been tested thoroughly on standard dataset for checking accuracy and consistency before applying to waterbody object extraction problem. Finally the accuracy of the data set is calculated using confusion matrix and kappa coefficient.

\subsection{Block Diagram}

The block diagram of the entire methodology has been shown in Figure 2 and Figure 3.

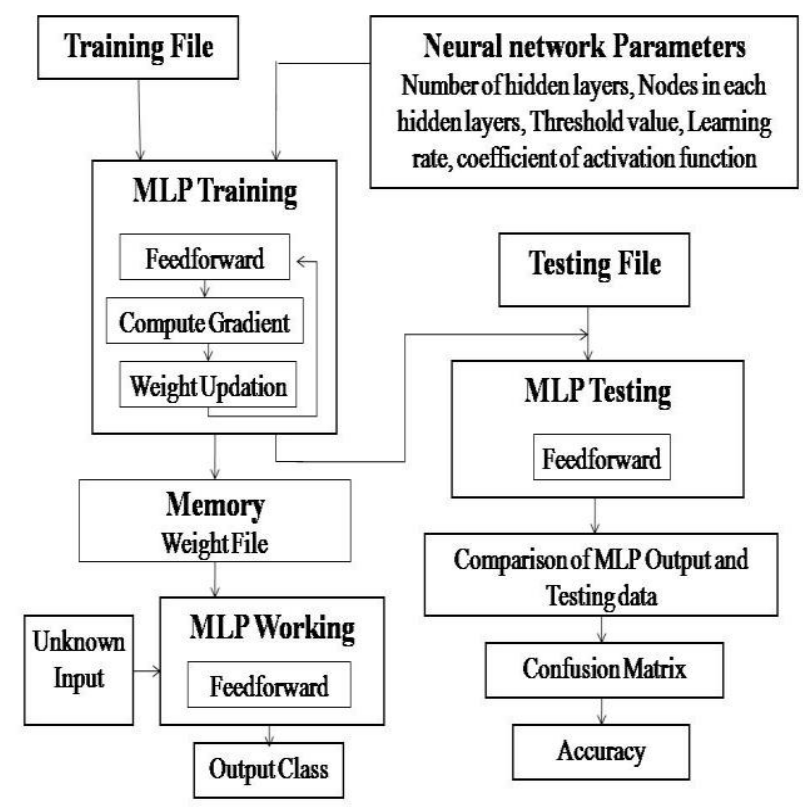

Figure 2: Block diagram of the MLP

Figure 2 shows block diagram of MLP consisting of various components i.e. MLP Training, MLP Testing and MLP Working blocks. It shows the flow of data and connectivity among various sub modules of MLP. Figure 3 shows the block diagram providing interface of MLP with image handling module. Image handling module read the satellite image; convert it into image data matrix. MLP takes each pixel from image data matrix and classify it whether it is waterbody or not. The extracted waterbody is then color coded to display.



Figure 3: Block diagram of the system for automatic extracting waterbody from satellite image

\subsection{Generation of Training and Testing Data}

The training dataset consists of prior knowledge of input patterns. Training and testing dataset have been generated from satellite images for two classes i.e. waterbody and nonwaterbody. Each pattern in training and testing dataset consists of three dimensional feature vector having Red, Green and Blue value corresponding to each pixel along with class label. The whole dataset generated by domain expert has been split into three sets of training and testing datasets where the ratio of number of patterns in training and testing dataset is 25:75 respectively. In each set, training dataset consists of 3000 patterns and testing dataset consists of 9000 patterns. These training and testing data sets are used for the training and testing purpose.

\subsection{Flow Charts}

Flowcharts corresponding to training and testing processes of MLP are given below. 


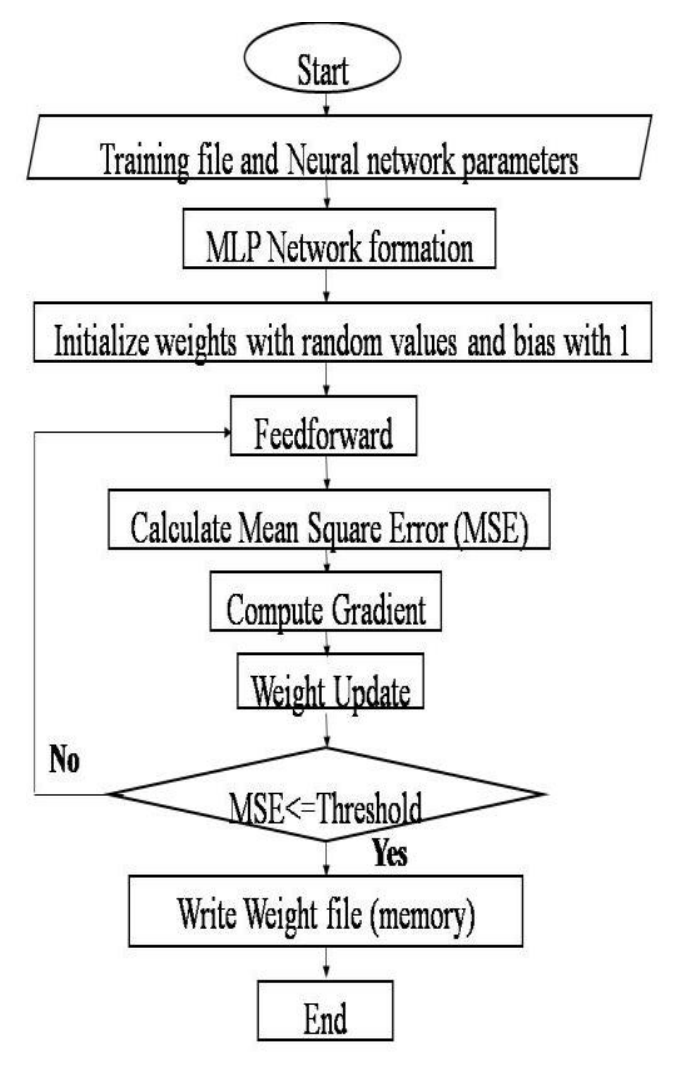

Figure 4: Flow Chart of training process

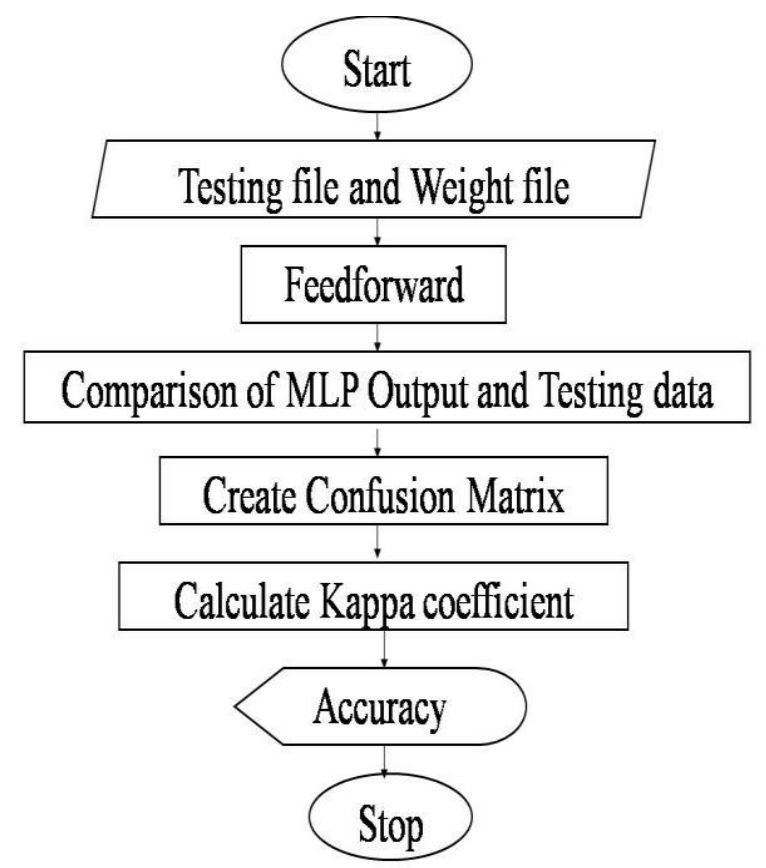

Figure 5: Flow Chart of testing process

\subsection{Algorithms}

The notations used in the algorithm are:

4.4.1 Algorithm for training MLP

\section{Notation Meaning}

$u_{l, j} \quad$ Output of the $j t h$ node in layer $l$

$w_{l, j, i} \quad$ Weight which connects the $i^{\text {th }}$ node in the layer $l-1$

to the $j^{\text {th }}$ node in layer $l$ $d_{j} \quad$ Desired response of the $j^{\text {th }}$ output node

$N_{l} \quad$ Number of nodes in the layer $l$

$L \quad$ Number of layers

$\mathrm{f}($.$) \quad Sigmoid nonlinearity function$

$e_{j} \quad$ Error at $j^{\text {th }}$ node

$G_{l, j} \quad$ Gradient of $j^{\text {th }}$ node of layer $l$

$\eta \quad$ Learning rate

1. Input: - Training Dataset

MLP Parameters (Number of hidden layers, Nodes in each hidden layer, Threshold value, Learning rate, Coefficient of activation function)

2. ErrorBackPropagation

a. Create MLP Network

b. Initialize weights to random values

c. Repeat for each pattern in training dataset Start FEEDFORWARD

For layer $=1$ to $\mathrm{L}$ do For node $=1$ to $N_{\text {layer }}$ do

End $\{$ FEED_FORWARD $\}$

$$
\begin{aligned}
u_{\text {layer }, \text { node }}= & f\left(\sum_{i=0}^{N_{\text {layer }-1}} w_{\text {layer }, \text { node }, i} *\right. \\
& \left.u_{\text {layer }-1, i}\right)
\end{aligned}
$$

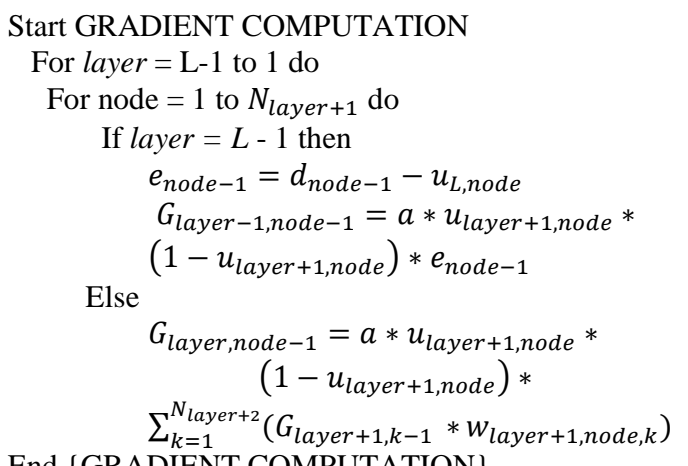

End $\{$ GRADIENT COMPUTATION $\}$

End \{WEIGHT UPDATION\}

$$
\begin{aligned}
& \text { Start WEIGHT UPDATION } \\
& \text { For layer }=0 \text { to L do } \\
& \text { For node }=1 \text { to } N_{\text {layer }+1} \text { do } \\
& \text { For count }=0 \text { to } N_{\text {layer }} \text { do } \\
& w_{\text {layer,count,node }}=w_{\text {layer,count,node }}+ \\
& \eta
\end{aligned}
$$

3. Write the weight (memory) file.

4.4.2 Algorithm for testing MLP

1. Input: - Testing Dataset

$$
\text { Weight file }
$$

2. Read MLP Parameters and Weights from weight file

3. Create MLP Network

4. Repeat for each pattern in testing dataset 4.1 Start FEEDFORWARD

For layer $=1$ to $\mathrm{L}$ do For node $=1$ to $N_{\text {layer }}$ do

$$
u_{\text {layer }, \text { node }}=f\left(\sum_{i=0}^{N_{\text {layer-1 }}} w_{\text {layer }, \text { node }, i} *\right.
$$$$
\left.u_{\text {layer }-1, i}\right)
$$

End \{FEED_FORWARD

4.2 Compare MLP output and testing dataset output.

5. Create Confusion matrix and Compute Kappa Coefficient. 6. Compute accuracy. 


\section{RESULTS AND DISCUSSION}

The results of the above experiment have been shown in this section. The results on standard datasets as well as the dataset for waterbody extraction have been shown in detail consisting of MLP architecture and other related parameters. The results on actual satellite images have also been given. Confusion matrix, kappa coefficient and line diagram showing results have also been given for better understanding.

\subsection{MLP Results for Standard Datasets}

Three standard datasets from UC Irvine Machine Learning Repository, Centre for Machine Learning and Intelligent Systems have been used for testing accuracy and consistency of MLP. Results for these standard datasets are shown below.

Table 1: Standard Datasets

\begin{tabular}{|c|c|c|c|}
\hline & Iris & $\begin{array}{c}\text { Image } \\
\text { Segmentation }\end{array}$ & Ionosphere \\
\hline $\begin{array}{c}\text { MLP } \\
\text { Architecture }\end{array}$ & $2(10,20)$ & $2(10,20)$ & $1(50)$ \\
\hline $\begin{array}{c}\text { Learning Rate } \\
(\eta)\end{array}$ & 0.2 & 0.2 & 0.2 \\
\hline $\begin{array}{c}\text { Number of } \\
\text { Attributes }\end{array}$ & 4 & 19 & 34 \\
\hline $\begin{array}{c}\text { Number of } \\
\text { Classes }\end{array}$ & 3 & 7 & 2 \\
\hline \begin{tabular}{c} 
Training MSE \\
\hline $\begin{array}{c}\text { Total Patterns } \\
\text { (Training) }\end{array}$
\end{tabular} 5.90E-6 & $2.63 \mathrm{E}-5$ & $3.64 \mathrm{E}-4$ \\
\hline $\begin{array}{c}\text { Training } \\
\text { Accuracy }\end{array}$ & $\mathbf{1 0 0 \%}$ & $\mathbf{1 0 0 \%}$ & 105 \\
\hline $\begin{array}{c}\text { Total Patterns } \\
\text { (Testing) }\end{array}$ & 105 & $\mathbf{9 8 . 0 9 \%}$ \\
\hline $\begin{array}{c}\text { Testing } \\
\text { Accuracy }\end{array}$ & $\mathbf{9 6 . 1 9 \%}$ & $\mathbf{9 3 . 0 4 \%}$ & $\mathbf{8 6 . 5 8 \%}$ \\
\hline
\end{tabular}

\subsection{MLP Results for Waterbody Object Extraction Dataset}

With respect to the problem i.e. automatic waterbody extraction from satellite images, three sets consisting of training and testing data in each and having two classes have been generated and used to train and test the MLP. The resultant weight file after completion of training of the MLP was then used to extract waterbody from satellite images. The results for training and testing accuracy for these three sets have been shown below.
Table 2: Waterbody Object Extraction Datasets

\begin{tabular}{|c|c|c|c|}
\hline & Set1 & Set2 & Set3 \\
\hline MLP Architecture & $1(80)$ & $1(80)$ & $1(80)$ \\
\hline Learning Rate $(\eta)$ & 0.2 & 0.2 & 0.2 \\
\hline Number of Attributes & 3 & 3 & 3 \\
\hline Number of Classes & 2 & 2 & 2 \\
\hline Training MSE & $1.50 \mathrm{E}-6$ & $1.91 \mathrm{E}-6$ & $3.45 \mathrm{E}-6$ \\
\hline Total Patterns (Training) & 3000 & 3000 & 3000 \\
\hline Training Accuracy & $\mathbf{9 9 . 8 3 \%}$ & $\mathbf{1 0 0 \%}$ & $\mathbf{9 9 . 6 \%}$ \\
\hline Total Patterns (Testing) & 9000 & 9000 & 9000 \\
\hline Testing Accuracy & $\mathbf{9 8 . 7 3 \%}$ & $\mathbf{9 9 . 2 2 \%}$ & $\mathbf{9 9 . 5 4 \%}$ \\
\hline
\end{tabular}

\subsection{Confusion Matrix for Waterbody} Object Extraction

The Confusion Matrix describing the accuracy for each set for waterbody extraction problem has been shown below.

\begin{tabular}{|c|c|c|c|c|}
\hline & & Class1 & Class2 & $\begin{array}{c}\text { Accuracy } \\
(\%)\end{array}$ \\
\hline \multirow{2}{*}{ Set1 Training } & Class1 & 249 & 4 & 98.42 \\
\hline & Class2 & 1 & 2746 & 99.96 \\
\hline \multirow{2}{*}{ Set1 Testing } & Class1 & 772 & 18 & 97.72 \\
\hline & Class2 & 6 & 8204 & 99.93 \\
\hline \multirow{2}{*}{ Set2 Training } & Class1 & 253 & 0 & 100 \\
\hline & Class2 & 3 & 2744 & 99.89 \\
\hline \multirow{2}{*}{ Set2 Testing } & Class1 & 792 & 27 & 96.70 \\
\hline & Class2 & 43 & 8138 & 99.47 \\
\hline \multirow{2}{*}{ Set3 Training } & Class1 & 249 & 4 & 98.42 \\
\hline & Class2 & 8 & 2739 & 99.71 \\
\hline \multirow{2}{*}{ Set3 Testing } & Class1 & 824 & 7 & 99.16 \\
\hline & Class2 & 34 & 8135 & 99.58 \\
\hline
\end{tabular}

Figure 6: Confusion Matrix of Waterbody Object Extraction Datasets 
5.4 Line Diagram showing accuracy of Waterbody Object Extraction

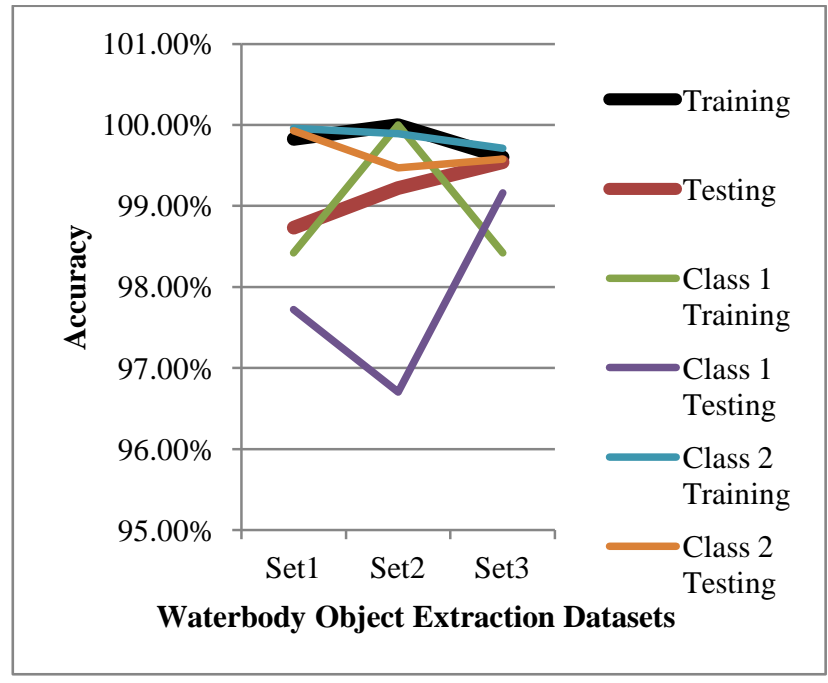

Figure 7: Training and testing accuracy

\subsection{Distribution of Training and Testing Dataset Patterns for Waterbody Object Extraction}

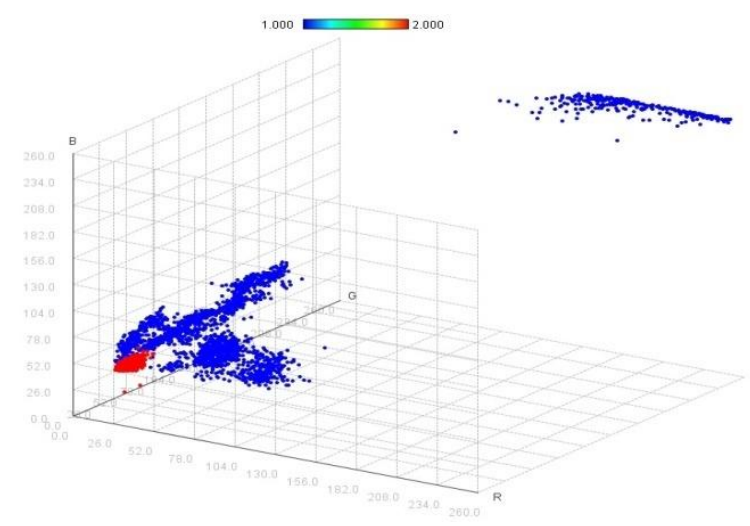

Figure 8: Training File (Red color: waterbody patterns)

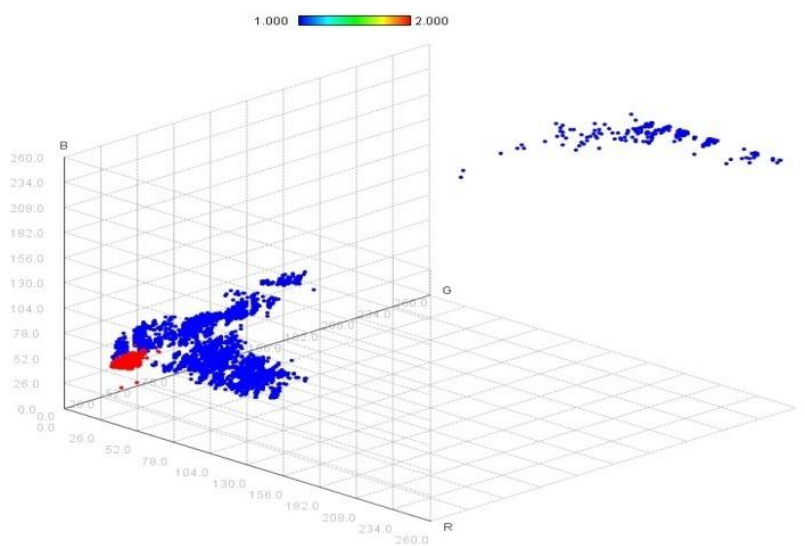

Figure 9: Testing File (Red color: waterbody patterns)
5.6 Kappa Coefficient

Table 3: Waterbody Object Extraction Datasets

\begin{tabular}{|c|c|c|}
\hline Datasets & $\begin{array}{c}\text { Kappa Coefficient } \\
\text { (training) }\end{array}$ & $\begin{array}{c}\text { Kappa Coefficient } \\
\text { (testing) }\end{array}$ \\
\hline Set1 & 0.9891 & 0.9832 \\
\hline Set2 & 0.9935 & 0.9533 \\
\hline Set3 & 0.9742 & 0.9732 \\
\hline
\end{tabular}

\subsection{Waterbody Object Extraction Output for Satellite Images}

Satellite images to be given as input to the system have been shown in Figure 10. The results obtained using this system has also been shown in this figure. Extracted waterbodies in these satellite images have been shown in blue color.

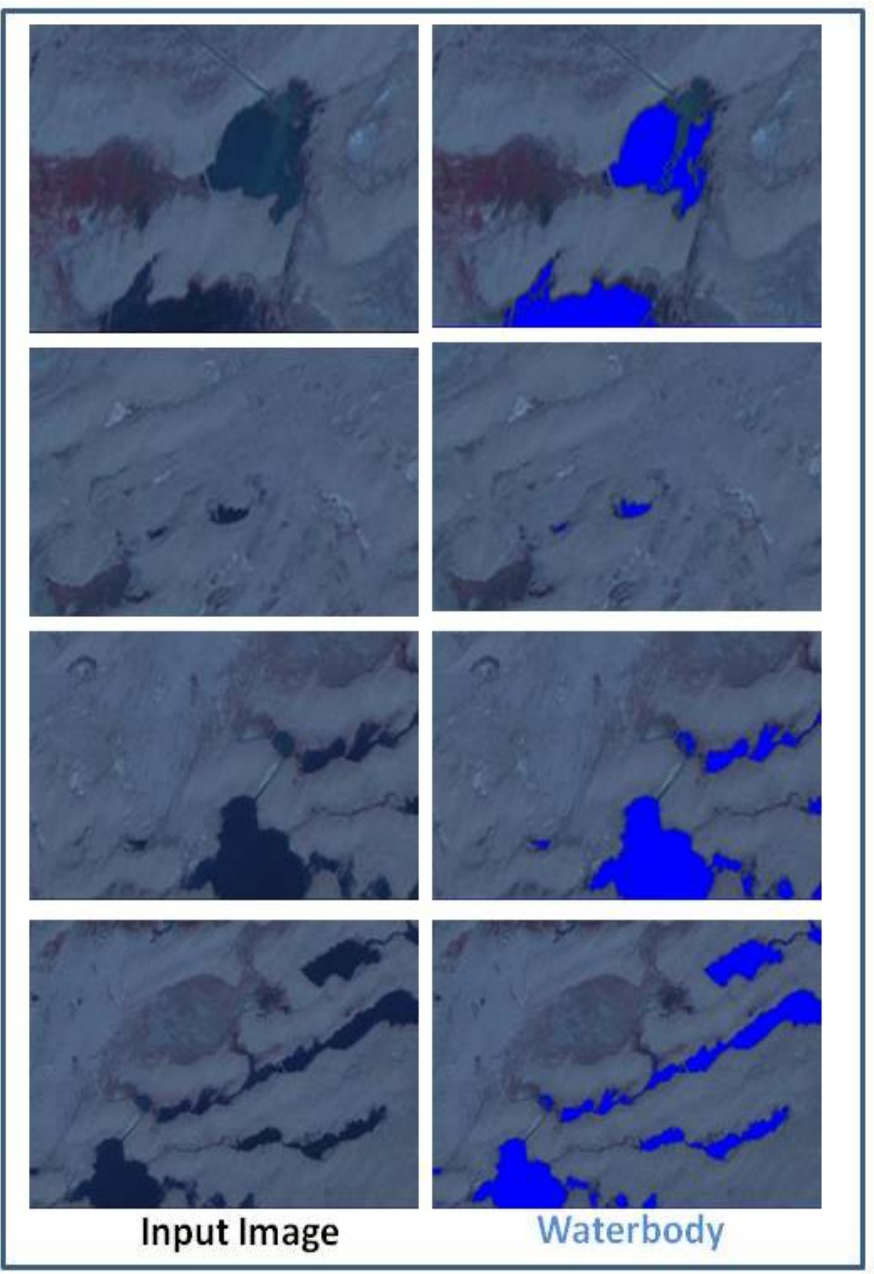

Figure 10: Waterbody Extraction Output 1 


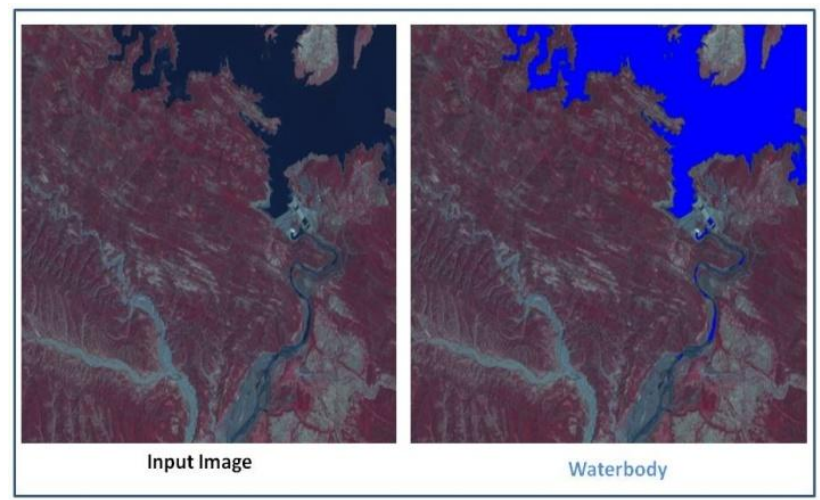

Figure 11: Waterbody Extraction Output 2

The results of the experiment shows that MLP is a very good algorithm for object recognition. Here it has given good results with accuracy more than $90 \%$ by extracting waterbody from satellite images in automatic manner. Once, training dataset has been generated by domain expert, then there is no need of domain expert. After training, MLP automatically extracts waterbody from satellite data. The training accuracy is almost $100 \%$ whereas testing accuracy is approx. $99 \%$. This shows the strength of MLP in image classification and object recognition. Distribution of class 1 and class 2 patterns in training and testing datasets shows their seperability from each other. Confusion matrix generated also shows that MLP can provide better results in such problems. One of the important point to be noted here is that the accuracy of MLP classifier heavily depends on the quality of training datasets and MLP architecture. Training datasets must be robust and consistent to give better accuracy.

\section{CONCLUSION}

The experiment performed has shown the strength of ANN to recognize various objects in an automatic manner. The experiment shows good accuracy for automatic extraction of waterbody from satellite data. It has also been observed that accuracy of MLP greatly depends upon the quality and robustness of training dataset. The accuracy also depends on the quality of training given to MLP. MLP can work in an effective and efficient manner for various satellite image classification problems based on the training data provided to it. Waterbody extraction is a two class problem. But MLP can be used for problems having more than two classes. Multi classes classification approach can be addressed using MLP in many ways. Some of them are as: fusion of various classification algorithms to increase accuracy and consistency of the results, fusion of two or more weight files i.e. two or more training of MLP with different architecture and parameters and training file for the same problem and then fusion of them, using fuzzy MLP etc. This experiment can be extended in future for approaching multi class classification problem using fusion based approach.

\section{REFERENCES}

[1] J. Vieira, F. M. Dias, A. Mota, Ding and W. Marchionini, "Neuro-Fuzzy Systems: A Survey ", A Study on Video Browsing Strategies, Technical Report, University of Maryland at College Park, 1997
[2] S. Haykin, "Neural Networks: A Comprehensive Foundation", NY: Macmillan, Phi Learning Pvt. Ltd., pp. 2, 1994.

[3] J. M. Zurada, "Introduction to Artificial Neural Systems", Boston: PWS Publishing Company, preface pp. 15, 1992.

[4] C. M. Bishop, "Neural Networks for Pattern Recognition", Oxford University Press, pp. 116-122, 1995.

[5] R. D. Reed, and R. J. Marks II, "Neural Smithing: Supervised Learning in Feedforward Artificial Neural Networks", Cambridge, MA: The MIT Press, ISBN 0262-181908, 1999.

[6] D. E. Rumelhart, G. E. Hinton, and R. J. Williams, "Learning internal representations by error propagation", ICS Report 8506,California University, San Diego, La Jolla, Institute for Cognitive Science, 1985.

[7] D. E. Rumelhart, and J. L. McClelland, "Parallel Distributed Processing: Explorations in the Microstructure of Cognition", Cambridge, MA: The MIT Press, vol. 1, pp. 318-362, 1986.

[8] V. Cherkassky and F. Mulier, "Learning from Data: Concepts, Theory and Methods", New York: Wiley, pp 156- 213,1998

[9] L. Bruzzone and S. B. Serpico, "Classification of imbalanced remote-sensing data by neural networks", Pattern Recognition Letters, vol. 18 no. 11, pp. 13231328, 1997.

[10] M. D. Emmerson and R. I. Damper, "Determining and Improving the Fault Tolerance of Multilayer Perceptrons in a Pattern-Recognition Application", IEEE Transactions on Neural Networks, vol. 4 no. 5, pp. 788-793, 1993.

[11] J. Yi and R. Prybutok, "A neural network model forecasting for prediction of maximum ozone concentration in an industrialized urban area", Environmental Pollution, vol. 92 no. 3, pp. 349-357, 1996.

[12] Marzban and G. J. Stumpf, "A neural network for tornado prediction based on Doppler radar derived attributes", Journal of Applied Meteorology, vol. 35, pp. 617-626, 1996.

[13] H. D. Navone and H. A. Ceccatto, "Predicting Indian monsoon rainfall: a neural network approach", Climate Dynamics, vol. 10, pp. 305-312, 1994.

[14] R. M. Welch, S. K. Sengupta, A. K. Goroch, P. Rabindra, N. Rangaraj and M. S. Navar, "Polar cloud classification using AVHRR imagery - an inter comparison of methods", Journal of Applied Meteorology, vol. 31, pp. 405-420, 1992.

[15] J. E. Peak and P. M. Tag, "Towards automated interpretation of satellite imagery for navy shipboard applications", Bulletin of the American Meteorological Society, vol. 73 no. 7, pp. 955-1008, 1992. 